\title{
Size distribution and waiting times for the avalanches of the Cell Network Model of Fracture
}

\author{
Gabriel Villalobos ${ }^{a *}$, Ferenc Kun ${ }^{c}$, Dorian L. Linero ${ }^{b}$, José D. Muñoz $^{a}$ \\ ${ }^{a}$ Simulation of Physical Systems Group, CeiBA-Complejidad, Department of Physics, \\ Universidad Nacional de Colombia, Crr 30 \# 45-03, Ed. 404, Of. 348, Bogota D.C., Colombia., \\ ${ }^{b}$ Analysis, Design and Materials Group, Department of Civil and Environmental Engineering, \\ Universidad Nacional de Colombia, Crr 30 \# 45-03, Ed. 404, Of. 348, Bogota D.C., Colombia., \\ ${ }^{c}$ Department of Theoretical Physics. University of Debrecen, H-4010 Debrecen, P.O.Box 5, Hungary.
}

November 2, 2018

\section{Abstract}

The Cell Network Model is a fracture model recently introduced that resembles the microscopical structure and drying process of the parenchymatous tissue of the Bamboo Guadua angustifolia. The model exhibits a power-law distribution of avalanche sizes, with exponent -3.0 when the breaking thresholds are randomly distributed with uniform probability density. Hereby we show that the same exponent also holds when the breaking thresholds obey a broad set of Weibull distributions, and that the humidity decrements between successive avalanches (the equivalent to waiting times for this model) follow in all cases an exponential distribution. Moreover, the fraction of remaining junctures shows an exponential decay in time. In addition, introducing partial breakings and cumulative damages induces a crossover behavior between two power-laws in the avalanche size histograms. This results support the idea that the Cell Network Model may be in the same universality class as the Random Fuse Model.

Statistical models of fracture Finite Element Method Computational mechanics of solids.

PACS 02.50.-r,05.90.+m,46.50.+a,62.20.F-, 62.20.M-

\section{Introduction}

The Cell Network Model of Fracture (CNMF [1]), is a two dimensional statistical model of fracture 22 inspired in the stress field caused by drying of the bamboo Guadua angustifolia 3, 4. At the parenchymatous tissue level, bamboos shrink during drying, causing the detaching of neighboring cells and the appearance of fractures. The CNMF models this tissue as an hexagonal array of cell elements (each of them made of six beams), fixed by angular springs and joined by brittle springs called the junctures. (Figure: 1 ).

\footnotetext{
${ }^{*}$ Corresponding author, gabrielvc@gmail.com
}

Shrinking forces -due to drying- acting along the elements distort the structure and cause breaking avalanches of the junctures among cells.

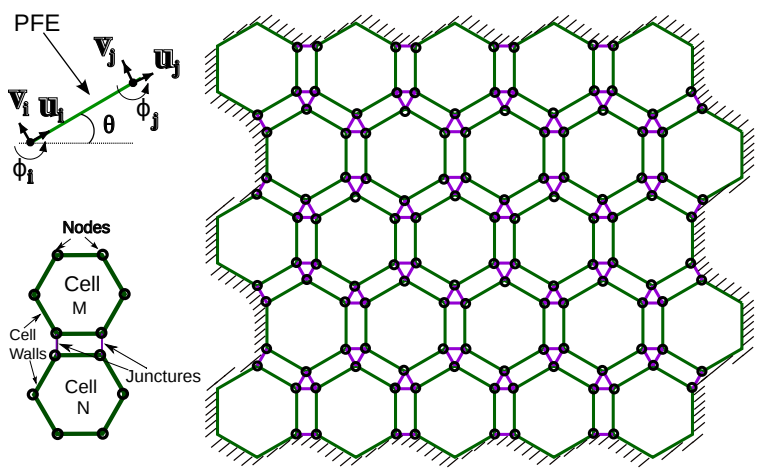

Figure 1: Cell Network Model of Fracture (CNMF). Upper left, The plane frame element spanning between the nodes $i$ and $j$, oriented by an angle $\theta$. Each node has two translational and one rotational degrees of freedom. Lower left. Two contiguous Cells. Right Structure of the CNMF. The hexagons represent the cells and the junctures are arranged into triangles. Hashing denote fixed boundary conditions. From [1] Not at scale.

Interestingly enough, when an homogeneous distribution of breaking thresholds of junctures and fixed Young modulii are used, the histogram of avalanche sizes of the CNMF shows power law behavior with an exponent of -2.93(8) ([1]). This is by all means equal to avalanche size distribution of the random fuse model, which shows a power law with exponent of -3 [5, 6]. The analytical solution of both models has been elusive.

One dimensional fiber bundle systems have provided models for the thoroughly study of critical phenomena. Universality, the effect of damage and the critical expo- 
nents have been found analytically (see [7, 8] and references therein.). The logical extension of those models to $2 \mathrm{D}$, the random fuse model, has allowed to investigate the fracture properties of biological materials, as in the case of brittle nacre, (9]), describing the toughness of the material by its microscopical architecture. Beam models similar to the CNMF has also been used to model fracture in concrete 10.

In the present paper, to characterize the path to global failure, we study both the distribution of humidity decrement among between consecutive avalanches (the analogue of waiting times for this model), as well as the fraction of intact fibers as function of the humidity decrement. This last quantity behaves as an order parameter for the system (see (Sec: 2)). Moreover, the universality of the CNMF is explored numerically by characterizing the histogram of avalanche sizes for two cases: homogeneous distributions of the juncture breaking thresholds with different widths and Weibull distributions of different shapes, (Sec: 3). Furthermore, in section (Sec: 4 ) a damage function is introduced as follows: Each time a stress threshold is reached, the stiffness is reduced by a constant factor, until the fiber completely breaks. The main results and comments are summarized in Sec (Sec: 5).

\section{Model}

The CNMF is a 2D statistical model of fracture that resembles the parenchymatous tissue of the bamboo Guadua augustifolia. It is composed by two kinds of structures: cell walls and junctures among cells. Six cell walls arrange themselves to form hexagons, thanks to a angular springs associated with the rotational degree of freedom. The cells are arranged like a honeycomb. The junctures are arranged in sets of three at the common corners of the cells, modeling the silica deposits that glue cells together. (Figure: 11 Each kind has a given fixed Young modulus for all its elements. Junctures are allowed to break, as a result of the brittle behavior of the silica, while cell walls are not.

As boundary conditions, all the border nodes are set fixed. The deformation of the system comes from shrinking forces acting on every cell wall and proportional to a global humidity loss parameter $\Delta h$. By means of a Finite Element Method, the resulting forces and deformations of all the elements are calculated.

The evolution of the system has three stages. Linear Elastic Shrinking: local shrinking forces due to humidity losses are applied to the cell walls. The differences between the local strain at the juncture and their individual thresholds are calculated. This step continues until at least one fiber would suffer an strain surpassing its threshold. Drying induced Breaking: By means of a zero finding algorithm, the exact humidity loss causing the first breaking is found. The broken element is removed from the struc- ture, changing the stiffness matrix in accordance. Nonlinear avalanche: The breaking of an element calls for a force redistribution over the whole structure. This redistribution may cause an avalanche of breaks. When the avalanche ends, the procedure re-stars from the first stage.

\subsection{Distributions of humidity decrement between consecutive avalanches}

The distribution of humidity decrements between successive avalanches provides a description of the path to the global failure of the system. This is the analog to the waiting times between avalanches of other models of fracture. Fig. (Figure: 2) shows the normalized histograms of humidity decrements for several values of the maximum humidity change allowed $\Delta h$. When the breaking process is driven until the end, the histograms can be fitted to an exponential, with a fitted humidity constant of $13.5(2)$, which is and indication of lack of correlation between successive avalanches.

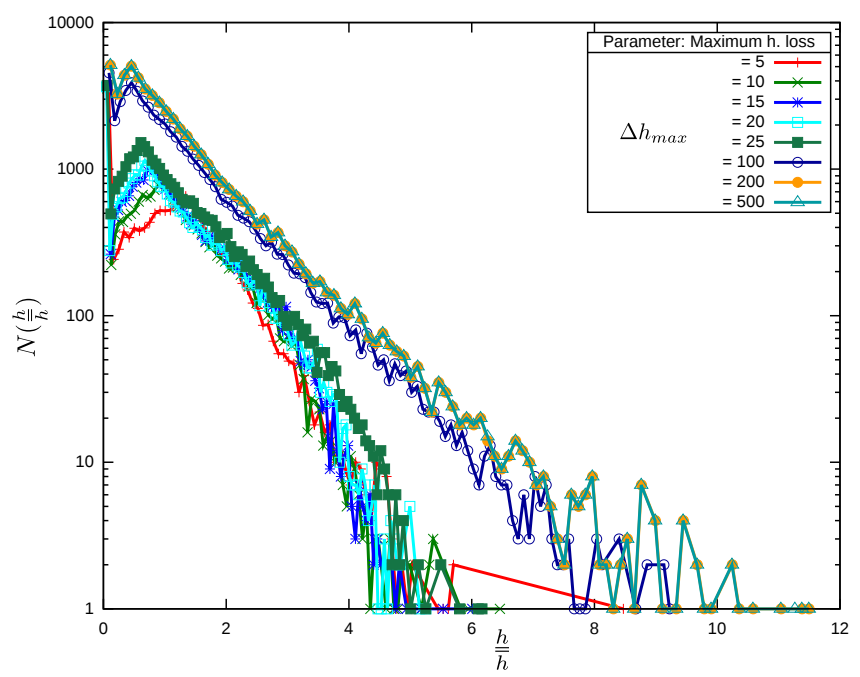

Figure 2: Humidity decrement between consecutive avalanches histogram. The parameter that defines a curve is the maximum humidity change ( $\Delta h$, proportional to Cell Wall strain, shown). The classes (horizontal axis) are the (normalized by the mean waiting humidity).

\subsection{Fraction of remaining junctures}

Let us consider the one-dimensional global load sharing fiber bundle model [8. Given $U(\sigma)$ as the fraction of remaining fibers at a given stress and $\sigma_{c}$ the critical stress causing the global breaking of the system. Thus, $U^{*}(\sigma)-U^{*}\left(\sigma_{c}\right)$ behaves as an order parameter. For the CNMF with homogeneous breaking thresholds at the juncture elements we obtain an exponential relaxation in the number of remaining junctures ((Figure: 3)). 


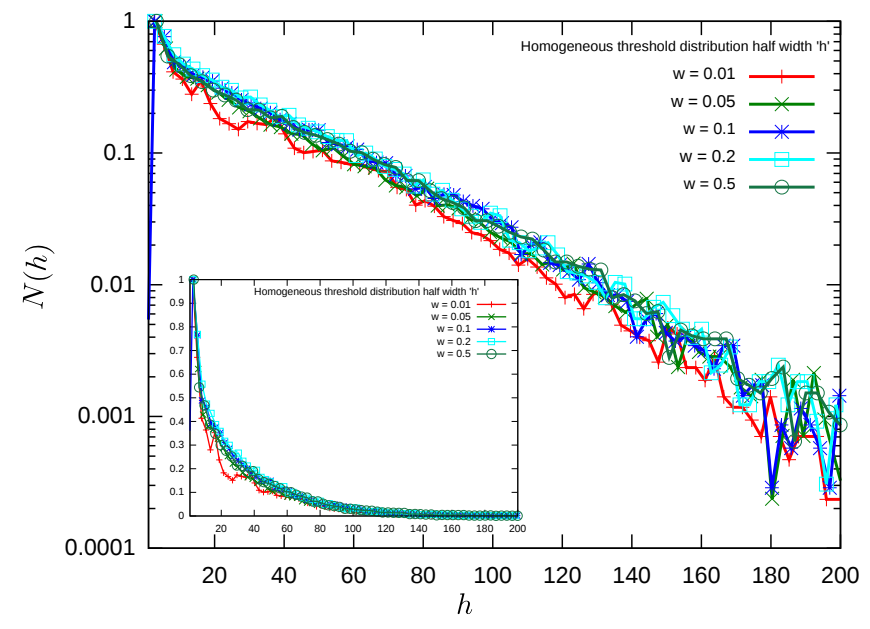

Figure 3: Number of intact fibers as function of the humidity change for several homogeneous distribution of the breaking threshold with different widths (semi log). Inset, linear axis.

This exponential behavior is independent of the system size. In (Figure: 4), the fraction of the population of intact fibers as function of humidity is shown for different sizes of the system. The horizontal axis was rescaled by means of a linear fit on the semi-log data. All histograms show an exponential decay.

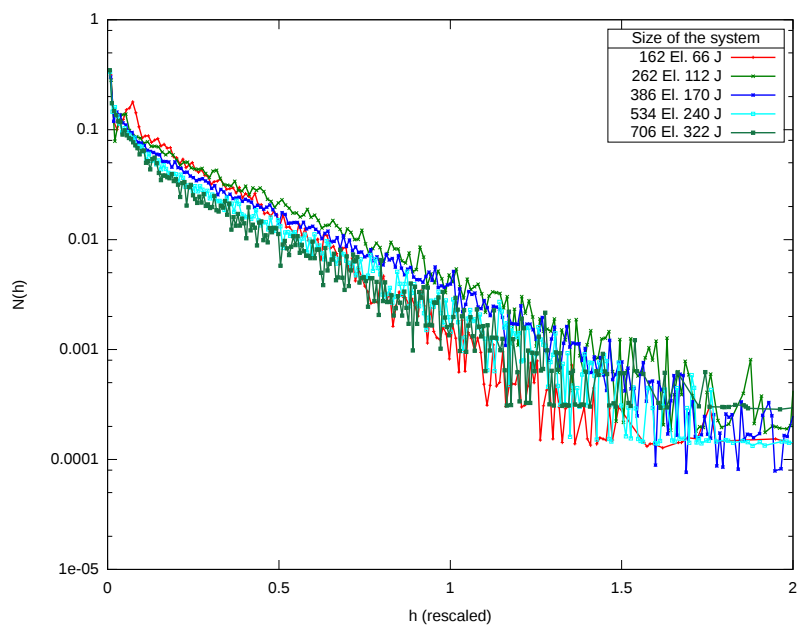

Figure 4: Scaled fraction of intact fibers as function of the humidity change (in units of max humidity difference) for different system sizes.

\section{Universality}

Fiber bundle models are universal in the sense that the breaking of the elements follows power law distribution of avalanche sizes irrespective of several system charac- teristics. For the CNMF we studied the distribution of avalanche sizes when the thresholds are generated either from flat distributions of several widths or from Weibull distributions with several characteristic parameters.

Flat distributions of the breaking thresholds, all centered at $0.35 \mathrm{EA}$ but with different widths (spanning on two orders of magnitude), show the same power-law distribution of avalanche sizes, with slopes around -3 (Fig. (Figure: 5p). The data shows that narrower distributions show larger fluctuations around this power law than wider ones.

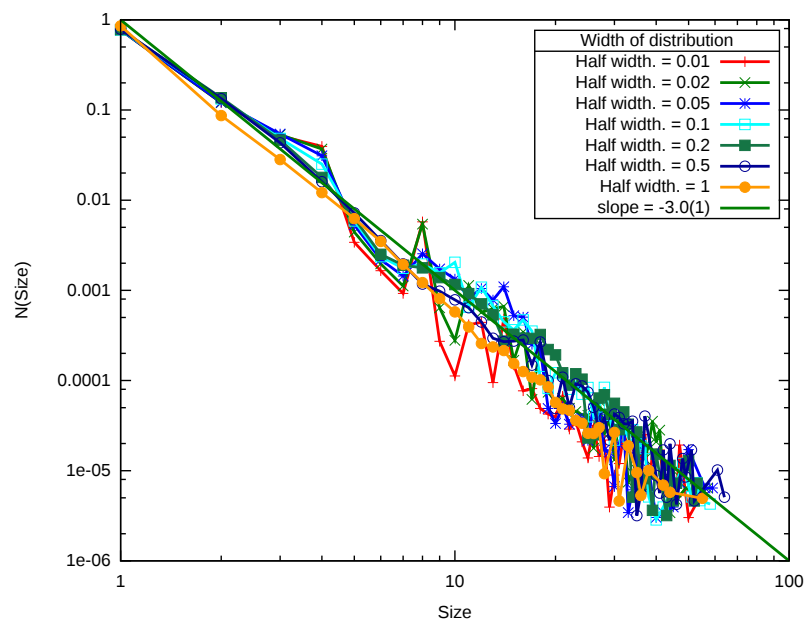

Figure 5: Histogram of avalanche sizes for several widths of the homogeneous threshold distribution centered at $0.35 E A$

The probability density function for the Weibull distribution function [11 is given by:

$$
f(x ; \lambda, k)=\left\{\begin{array}{cc}
\frac{k}{\lambda}\left(\frac{k}{\lambda}\right)^{k-1} e^{-(x / \lambda)^{k}} & x \geq 0 \\
0 & x<0
\end{array}\right.
$$

where $k$ and $\lambda$ are free parameters. It is commonly used to describe the breaking thresholds of fibers by fixing $\lambda=1$ and changing $k$, which is the main parameter controlling the distribution shape. When we use this distribution for the breaking thresholds at the junctures, the histogram of avalanche sizes shows also a power law behavior, with an exponent close to -2.9 for small values of $\mathrm{k}$. For larger values the exponential cutoff is more pronounced. (Figure: 6).

The humidity decrements between consecutive avalanches (that is, the waiting times) distributes like an exponential, also when the thresholds follow a Weibull distribution ((Figure: 7)). The characteristic time for $k=5$ is $16.1(8)$, very close to the one we gathered for flat distributions of the braking thresholds. 


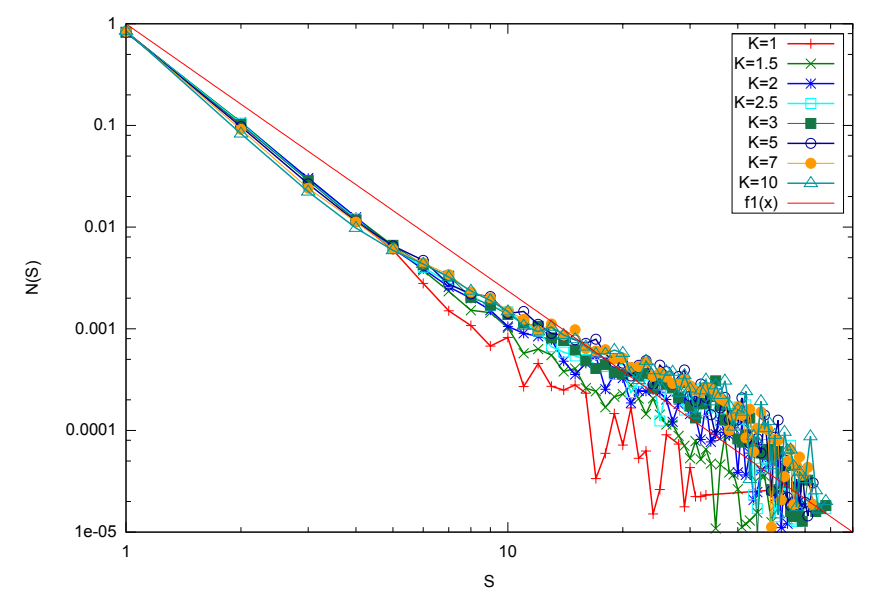

Figure 6: Histogram of avalanche sizes for several Weibull distributions of the breaking thresholds, with values of the shape parameters $k$ between 1 and 10. The straight line corresponds to an slope of -2.9 .

\section{Damage}

In order to introduce a degradation for the juncture elements we reduce the Young modulus of the juncture elements by a damage factor $0<a<1$ each time the juncture fails. When the element has suffered a maximum number of failures $k_{\max }$, it is assumed to be broken and is removed from the structure.

When a small degradation is introduced $(a=0.1)$, the power law distribution of avalanche sizes seems to exhibit a crossover from an exponent -3 to an exponent -2 at sizes around 8 (Fig. (Figure: 8)). This may indicate that the remaining elasticity helps to sustain the structure. However, more statistics and larger system sizes are required to clarify this point. Even smaller values of $a$ (not shown) cause the maximum humidity loss (and therefore the forces on the elements) to be much larger, creating numerical instabilities that end into poor statistics.

\section{Conclusions}

The numerical evidence of this work indicates that the avalanche sizes for the Cell Network Model of Fracture distribute as a power law with exponent -3.0 , for any broad distribution of the braking thresholds, either if they are flat or Weibull distributed.

The distribution of waiting times show an exponential decay for all system sizes evaluated and all tested disorder distributions of breaking thresholds. Even the characteristic times are similar for all of them. In our opinion, this is related to the fact that the most common failure mode for the system is the softening of the sample by displacements that would violate the boundary conditions.

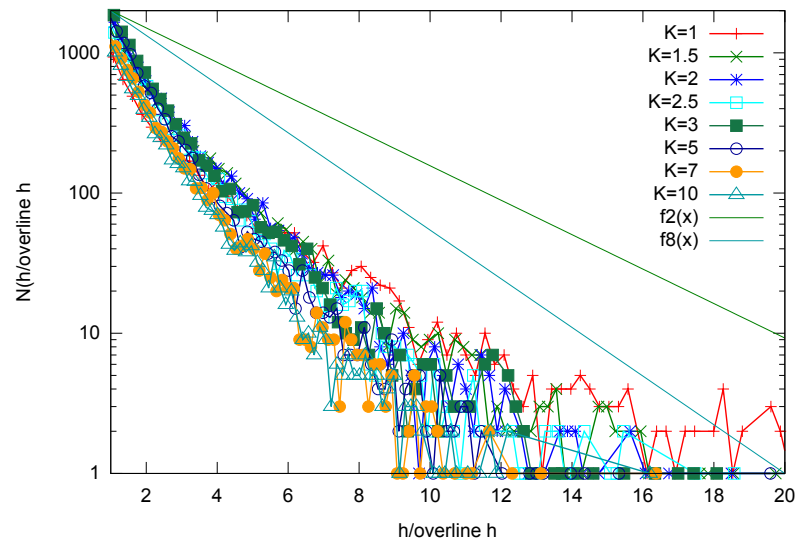

Figure 7: Histogram of humidity increments between successive avalanches for several Weibull distributions of the breaking thresholds, with $k$ between 1 and 10 . The blue line corresponds to a fit of the series of shape $k=10$, with slope -2.1 . The green line to that for $k=3$, with slope $-2.4$

Acknowledgments: We thank COLCIENCIAS ("Convocatoria Doctorados Nacionales 2008"), Centro de Estudios Interdisciplinarios Básicos y Aplicados en Complejidad-CeiBA - Complejidad and Universidad $\mathrm{Na}$ cional de Colombia for financial support. We also thank Professor Jorge A. Montoya and Professor Caori P. Takeuchi for enlightening discussions in the field of Guadua drying.

\section{References}

[1] G. Villalobos, D. L. Linero, J. D. Muñoz, A statistical model of fracture for a $2 \mathrm{~d}$ hexagonal mesh: the cell network model of fracture for the bamboo guadua angustifolia, Computer Physics Communicationsdoi : http://dx.doi.org/10.1016/j.cpc.2010.06.015. URL arXiv: 1002.3417

[2] M. J. Alava, P. K. V. V. Nukala, S. Zapperi, Statistical models of fracture, Advances in Physics 55 (2006) 349. URL arXiv . org: cond-mat/0609650

[3] J. A. Montoya Arango, Trocknungsverfahren furr die bambusart guadua angustifolia unter tropischen bedingungen, Ph.D. thesis, Universitat Hamburg (2006).

[4] C. Takeuchi, J. F. Rivera, Structural behaviour of braced guadua frames., in: 11th International Conference on Non-conventional Materials and Technologies NOCMAT, 2009.

[5] S. Pradhan, A. Hansen, P. C. Hemmer, Crossover behavior in failure avalanches, Phys. Rev. E 74 (1) (2006) 016122. doi:10.1103/PhysRevE.74.016122. 


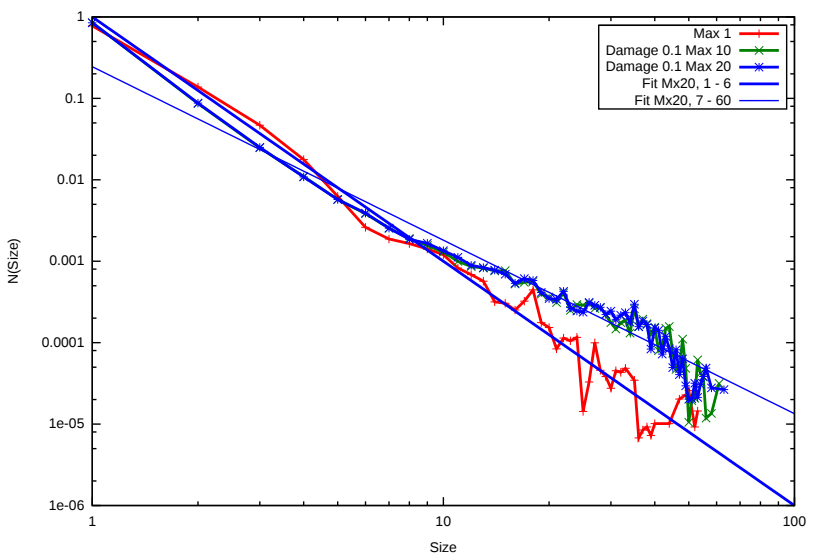

Figure 8: Histogram of avalanche sizes for damage parameter $a=0.1$ and several maximal numbers of failures, $k_{\max }$. The bolder line (slope $-2.98(7)$ fits for avalanche sizes between 1 and 7, while the thinner line (slope -2.1(1)) fits for avalanche sizes between 7 and 60 .

[6] A. Hansen, P. Hemmer, Phys. Lett. A. 184 (394).

[7] F. Kun, F. Raischel, R. C. Hidalgo, H. J. Herrmann, Modelling Critical and Catastrophic Phenomena in Geoscience, 2006, Ch. Extensions of fiber bundle models. doi:10.1007/b11766995.

URL http://www.ica1.uni-stuttgart.de/ hans/ fibres.html

[8] S. Pradhan, A. Hansen, B. K. Chakrabarti, Failure processes in elastic fiber bundles, Rev. Mod. Phys. 82 (1) (2010) 499-555. doi:10.1103/RevModPhys. 82. 499.

[9] P. K. V. V. Nukala, S. Šimunović, Statistical physics models for nacre fracture simulation, Phys. Rev. E 72 (4) (2005) 041919. doi:10.1103/PhysRevE.72. 041919 .

[10] S. A. Galindo Torres, J. D. Muñoz Castaño, Simulation of the hydraulic fracture process in two dimensions using a discrete element method, Phys. Rev. E 75 (6) (2007) 066109. doi:10.1103/PhysRevE.75. 066109 .

[11] W. Weibull, A statistical distribution function of wide applicability, ASME Journal of Applied Mechanics (1951) $293-297$.

URL http://www.barringer1.com/wa_files/ Weibull-ASME-Paper-1951.pdf 